\title{
Droplet vertical sizing in warm clouds using passive optical measurements from a satellite
}

\author{
A. Kokhanovsky and V. V. Rozanov \\ Institute of Environmental Physics, University of Bremen, 28334, Bremen, Germany \\ Correspondence to: A. Kokhanovsky (alexk@iup.physik.uni-bremen.de) \\ Received: 4 July 2011 - Published in Atmos. Meas. Tech. Discuss.: 29 August 2011 \\ Revised: 14 December 2011 - Accepted: 19 January 2012 - Published: 7 March 2012
}

\begin{abstract}
In this paper a new algorithm for the determination of the vertical distribution of the droplet effective radius in shallow warm clouds is proposed. The method is based on the fact that the spectral top-of-atmosphere reflectance in the near IR spectral range depends on the vertical profile of the effective radius of droplets. The retrieval is based on the optimal estimation method and direct radiative transfer calculations of respective weighting functions. The applications of the method both to synthetic and satellite data are presented. An important feature of the method is the fact that the cloud optical thickness and cloud effective radius are found using the standard homogeneous cloud retrieval and then the retrievals are improved assuming the type of the droplet effective radius profile.
\end{abstract}

\section{Introduction}

The determination of the effective radius (ER) of particles (droplets, crystals) in clouds using spaceborne observations is a very important remote sensing task. This is mostly due to the fact that clouds are major regulators of climate and their global properties are poorly understood. Usually the effective radius is derived using bi-spectral measurements under assumption that the vertical inhomogeneity of a cloud layer can be neglected. The channel in the visible is used to find the cloud optical thickness (COT) and the channel in the near-infrared is used to find the effective radius. The retrieved radius depends on the used satellite channel because clouds are vertically inhomogeneous and the penetration depth into the cloud depends on the wavelength (Platnick, 2000; Kokhanovsky, 2006; Zhang et al., 2010).
As a matter of fact, the inversion problem must be formulated in another way as far as the cloud droplet optical sizing of vertically inhomogeneous clouds is concerned. Namely, not the effective radius for the whole cloud layer must be sought but rather the corresponding vertical profile are to be derived from available satellite data (e.g. from multispectral measurements). Some results in this direction have already been reported (Chang and Li, 2002). In particular, the lookup table technique (LUT) was used. LUTs have been calculated for various vertical profiles of the effective radius. It was found that measurements at 1.24, 1.65, 2.1, and 3.75 micrometers are sufficient to find the linear profile of the effective radius. Later the same authors generalized their approach for nonlinear profiles (Chang and Li, 2003).

The task of this work is to develop an algorithm to retrieve the vertical profile of the droplet effective radius, which is based not on the generation of LUTs but rather it uses direct radiative transfer calculations in the retrieval process. The most important advantage of such an approach is that one does not need to create LUTs in advance and, therefore, the algorithm is more flexible with respect to change in a priori assumptions, parameters of instruments, etc. The retrieval is performed in the framework of the optimal estimation technique (Rodgers, 2008).

The structure of the paper is as follows. In the next section we address some theoretical background related to the calculations of the functional derivatives of the cloud reflectance with respect to the vertical profile of effective radius. Then the retrieval algorithm is introduced. Its performance is studied using a number of numerical experiments. Finally, the algorithm is applied to the satellite data. 


\section{The functional derivative of reflectance with respect to the vertical profile of droplet sizes}

\subsection{Main equations}

The variation of the spectral cloud reflectance $R(\lambda)$ at the wavelength $\lambda$ with respect to the variation $\delta a_{\mathrm{ef}}(z)$ of the effective radius profile $a_{\mathrm{ef}}(z)$ and the variation $\delta N(z)$ of the particle number concentration profile $N(z)$ along the vertical coordinate $z$ can be presented in the following form:

$\delta R(\lambda)=\int_{z_{1}}^{z_{2}} W_{a}(z, \lambda) \frac{\delta a_{\mathrm{ef}}(z)}{a_{\mathrm{ef}}(z)} \mathrm{d} z+\int_{z_{1}}^{z_{2}} W_{N}(z, \lambda) \frac{\delta N(z)}{N(z)} d z$,

where we neglected nonlinear terms and

$$
W_{a}(z, \lambda)=\frac{\delta R(\lambda)}{\delta \ln a_{\mathrm{ef}}(z)}, \quad W_{N}(z, \lambda)=\frac{\delta R(\lambda)}{\delta \ln N(z)}
$$

are the logarithmic functional derivatives of the function $R(\lambda)$ with respect to the functions $a_{\mathrm{ef}}(z)$ and $N(z)$, respectively. Here $z$ is the vertical coordinate and it is assumed that properties of clouds do not change in the horizontal plane. The cloud is positioned between levels $z_{1}$ (bottom) and $z_{2}$ (top). We will assume that although $a_{\mathrm{ef}}$ depends on the altitude within the cloud, this is not the case for the effective variance $v_{\mathrm{ef}}$ of the droplet size distribution $f(a, z)$, which is assumed to be known in advance and not dependent on $z$. This allows us to consider only the derivative of the reflectance to one parameter of the particle size distribution, namely, $a_{\mathrm{ef}}$. It is known (Kokhanovsky, 2006) that the cloud radiative properties only slightly depend on the effective variance and they are largely determined by the effective radius of droplets. Therefore, our approach is well justified.

The functions $W_{a}(z, \lambda) / a_{\mathrm{ef}}(z)$ and $W_{N}(z, \lambda) / N(z)$ are called the weighting functions (WF) in the inverse problem theory (see, e.g. Ustinov, 2007). The derivatives $W_{a}(z, \lambda)$ and $W_{N}(z, \lambda)$ can be interpreted as WFs with respect to the relative variation of the effective radius and number concentration profiles, respectively.

Although the function $W(z, \lambda)$ and other related functions depend on the profiles $a_{\mathrm{ef}}(z)$ and $N(z)$, we will not denote this dependence explicitly for the sake of simplicity. The concept of WF is used to understand how the variation of the profile $\delta a_{\mathrm{ef}}(z)$ is related to the variation of the reflectance $\delta R$ as measured by an optical instrument. The function $W_{a}(z, \lambda)$ $\left(W_{N}(z, \lambda)\right)$ has a dimension of inverse length and shows how important the variation of the profile $a_{\mathrm{ef}}(z)(N(z))$ is at a given level $z$ with respect to the modification of the cloud reflectance. For instance, one may neglect the influence of the droplet size vertical profile on the cloud reflectance at a given wavelength $\lambda$ and level $z$ in the cloud if $W_{a}(z, \lambda) \rightarrow 0$.

Introducing the relative variation of reflectance

$$
y(\lambda)=\frac{\delta R(\lambda)}{R(\lambda)}
$$

we can rewrite Eq. (1) in the following equivalent form:

$$
y(\lambda)=\int_{z_{1}}^{z_{2}} K_{a}(z, \lambda) \frac{\delta a_{\mathrm{ef}}(z)}{a_{\mathrm{ef}}(z)} d z+\int_{z_{1}}^{z_{2}} K_{N}(z, \lambda) \frac{\delta N(z)}{N(z)} d z,
$$

where we introduced the logarithmic functional derivatives (LFD) of $\ln R(\lambda)$ :

$$
K_{a}(z, \lambda)=\frac{\delta \ln R(\lambda)}{\delta \ln a_{\mathrm{ef}}(z)}, \quad K_{N}(z, \lambda)=\frac{\delta \ln R(\lambda)}{\delta \ln N(z)} .
$$

The study of the LFDs presented here is of importance for the development of cloud profiling techniques using ground, airborne, and satellite measurements. It shows how the relative variation of the effective radius profile $p_{a}(z)=\frac{\delta a_{\mathrm{e}}(z)}{a_{\mathrm{ef}}(z)}$ and the number concentration profile $p_{N}(z)=\frac{\delta N(z)}{N(z)}$ along the vertical coordinate in the region $\left[z_{1}, z_{2}\right]$ are related to the relative variation of reflectance $y(\lambda)$ :

$y(\lambda)=\int_{z_{1}}^{z_{2}} K_{a}(z, \lambda) p_{a}(z) \mathrm{d} z+\int_{z_{1}}^{z_{2}} K_{N}(z, \lambda) p_{N}(z) \mathrm{d} z$.

It is surprising, taking into account the explosion of scientific publications in the general area of cloud remote sensing, how little attention was given to studies of the functional derivatives described above. Note that the weighting functions considered here differ from those introduced by Platnick (2000). In the case considered by him, the weighting function $w\left(\tau, \tau_{0}\right)$ was defined in such a way that the retrieved effective radius $\bar{a}$ for an inhomogeneous cloud as determined from a single IR channel is related to the corresponding profile $a_{\mathrm{ef}}(\tau)$ as

$\bar{a}=\int_{0}^{\tau_{\mathrm{cl}}} a_{\mathrm{ef}}(\tau) w\left(\tau, \tau_{\mathrm{cl}}\right) d \tau$.

Here $\tau$ is the optical depth inside the cloud and $\tau_{\mathrm{cl}}$ is the cloud optical thickness. Recently such an approach was explored by Nakajima et al. (2010a, 2010b) for studies of droplet growth as detected from a satellite.

\subsection{The retrieval for the special case of known cloud optical thickness}

The cloud optical thickness can be estimated independently or before the retrieval of cloud droplet radius profile (e.g. from the measurements in the visible, which are not very sensitive to the droplet size profile). Clearly, if it is assumed that COT is known, the profiles $N(z)$ and $a_{\mathrm{ef}}(z)$ become interrelated and we can re-formulate our problem in such a way that not two but just one profile $\left(a_{\mathrm{ef}}(z)\right)$ is sought. Let us demonstrate it firstly using the geometrical optics approximation (Kokhanovsky, 2006) for the extinction cross section $C_{\text {ext }}(a)$ of water droplets. 
The cloud optical thickness is defined as

$$
\tau=\int_{z_{1}}^{z_{2}} \kappa_{\operatorname{ext}}(z) \mathrm{d} z
$$

where $\kappa_{\text {ext }}(z)=N(z)<C_{\text {ext }}(z)>$ is the cloud extinction coefficient and

$$
<C_{\text {ext }}(z)>=\int_{a_{1}}^{a_{2}} C_{\text {ext }}(a) f(a, z) \mathrm{d} a
$$

is the average extinction cross section of droplets having the size distribution $f(a, z)$. It is assumed that the size of droplets $a$ in a cloud varies from $a_{1}$ to $a_{2}$.

One derives in a good approximation valid as $\lambda / a \rightarrow$ 0 (van de Hulst, 1981; Kokhanovsky, 2006): $C_{\text {ext }}(a)=$ $2 \pi a^{2}$ and, therefore, $<C_{\mathrm{ext}}>=2 \pi<a^{2}>$, where $<a^{2}>=$ $\int_{a_{1}}^{a_{2}} a^{2} f(a, z) \mathrm{d} a$. We will assume in this work that the droplet size distribution is given by the following formula:

$f(a, z)=B(z) a^{\mu} \exp \left(-\mu a / a_{0}(z)\right)$,

where $B(z)=\mu^{\mu+1} / a_{0}^{\mu+1}(z) / \Gamma(\mu+1), \Gamma(\mu+1)$ is the gamma function, $a_{0}(z)$ is the mode radius and $\mu$ is the halfwidth parameter assumed to be equal 6.0.

It follows for the distribution given by Eq. (10) (Kokhanovsky, 2006):

$$
\begin{gathered}
a_{\mathrm{ef}}(z)=\left\langle a^{3}(z)\right\rangle /\left\langle a^{2}(z)\right\rangle=a_{0}(z)\left(1+\frac{3}{\mu}\right), \\
\left\langle a^{2}(z)\right\rangle=\beta a_{\mathrm{ef}}^{2}(z),
\end{gathered}
$$

where $\beta=(\mu+1)(\mu+2) /(\mu+3)(\mu+3)$ at integer $\mu$. Therefore, Eq. (8) results in:

$\tau=2 \pi \beta \int_{z_{1}}^{z_{2}} N(z) a_{\mathrm{ef}}^{2}(z) \mathrm{d} z$

This equation shows that, if $\tau$ is known in advance, then the profiles of the effective radius and the number droplet concentration are not independent. In particular, varying Eq. (12) with respect to $N(z)$ and $a_{\mathrm{ef}}(z)$, and taking into account that $\delta \tau=0$, we have

$$
\int_{z_{1}}^{z_{2}} a_{\mathrm{ef}}^{2}(z) \delta N(z) \mathrm{d} z+2 \int_{z_{1}}^{z_{2}} N(z) a_{\mathrm{ef}}(z) \delta a_{\mathrm{ef}}(z) \mathrm{d} z=0 .
$$

Assuming further that the relative variation $p_{N}=$ $\delta N(z) / N(z)$ does not depend on the vertical coordinate (e.g. the constant profile of the droplet number distribution as discussed by Miles et al. (2000) for shallow marine clouds), we obtain from Eq. (13):

$$
p_{N}=-\frac{4 \pi \beta}{\tau} \int_{z_{1}}^{z_{2}} N(z) a_{\mathrm{ef}}^{2}(z) p_{a}(z) \mathrm{d} z
$$

Thus, the relative variation of the particle number density can be expressed via the relative variation of the effective radius. It also means that we reduce the problem, where two unknown functions $\left(a_{\mathrm{ef}}(z), N(z)\right)$ are involved, to a simpler problem of finding just the profile of the effective droplet radius. In a more general case, when the geometrical optics approximation $(\lambda / a \rightarrow 0)$ is not valid, it follows: $p_{N}=\Lambda\left[p_{a}(z)\right]$, where $\Lambda$ is a linear integral transformation of $p_{a}(z)$ similar (but not identical) to Eq. (14). In particular, we derive in this more general case (see Appendix):

$p_{N} \equiv \Lambda\left[p_{a}(z)\right]=-\frac{1}{\tau_{0}} \int_{z_{1}}^{z_{2}} \frac{\partial \kappa_{\mathrm{ext}}\left(z, \lambda_{0}\right)}{\partial \ln a_{\mathrm{ef}}(z)} p_{a}(z) d z$,

where $\tau_{0}$ is the cloud optical thickness at the wavelength $\lambda_{0}$ and $\kappa_{\text {ext }}\left(z, \lambda_{0}\right)$ is the extinction coefficient at the same wavelength.

Substituting $p_{N}$ given by Eq. (15) into Eq. (6) instead of $p_{N}(z)$, and introducing the integrated LFD with respect to the number droplet concentration:

$K_{N}(\lambda)=\int_{z_{1}}^{z_{2}} K_{N}(z, \lambda) \mathrm{d} z$

we have:

$y(\lambda)=\int_{z_{1}}^{z_{2}} \tilde{K}_{a}(z, \lambda) p_{a}(z) \mathrm{d} z$

where

$\tilde{K}_{a}(z, \lambda)=K_{a}(z, \lambda)-M_{a}(z, \lambda)$

and

$M(z, \lambda)=\frac{K_{N}(\lambda)}{\tau_{0}} \frac{\partial \kappa_{\mathrm{ext}}\left(z, \lambda_{0}\right)}{\partial \ln a_{\mathrm{ef}}(z)}$.

Summarizing, the relation between functions $K_{a}(z, \lambda)$ and $\tilde{K}_{a}(z, \lambda)$ follows from the assumption that COT is known in advance and, therefore, profiles $a_{\mathrm{ef}}(z)$ and $N(z)$ are inter-related.

\subsection{Numerical results}

The general technique for the numerical calculations of LFDs using solutions of forward and adjoint radiative transfer equations is given by Rozanov and Rozanov (2007). The technique is incorporated in the software package SCIATRAN (Rozanov et al., 2012). We will use SCIATRAN for studies of the LFDs. In particular, in this section we will consider the main properties of the LFDs with respect to the vertical profile of droplet effective radius using SCIATRAN calculations. We assume that the cloud optical thickness is known fairly well (e.g. from the application standard cloud retrieval algorithm developed for homogeneous clouds 
(Kokhanovsky, 2006)). It is known that vertical inhomogeneity does not impact COT retrievals considerably because information on COT comes primarily from the visible channel, where the cloud single scattering albedo is very close to 1.0 (Sobolev, 1975).

To perform numerical calculations, we will replace the integral in Eq. (17) by the sum:

$y(\lambda)=\sum_{k=1}^{n} S\left(z_{k}, \lambda\right) p_{a}\left(z_{k}\right)$

where

$S\left(z_{k}, \lambda\right)=\tilde{K}_{a}\left(z_{k}, \lambda\right) w_{k} \Delta z_{k}$,

$\Delta z_{k}$ is the thickness of the sub-layer inside the cloud and $w_{k}$ is the corresponding weight taken to be equal 1.0 in this paper, except 0.5 for the first and last weight (the trapezoidal rule for the equidistant grid). For instance, assuming that $p_{a}\left(z_{k}\right)=1$ (i.e. the relative variation of the effective radius is equal to $100 \%$ at all levels), one can derive $y(\lambda)$ just summing up $S\left(z_{k}, \lambda\right)$ at all levels.

The results of numerical calculations of $S\left(z_{k}, \lambda\right)$ are presented in Figs. 1-3. Let us discuss them now. The absolute value of $S\left(z_{k}\right)$ is presented in Fig. 1a-f for channels $0.865,1.24,1.6,2.13,3.7$ micrometers and several values of COT $(1,5,10,20,50,100)$ for vertically homogeneous clouds with the effective radius 12 micrometers at the solar zenith angle (SZA) 60 degrees and the nadir observation (viewing zenith angle $\mathrm{VZA}=0$ degrees). Thermal emission is taken into account for calculations at $3.7 \mu \mathrm{m}$. Note that in all cases LFDs are negative and we plotted the absolute values for the convenience. The negative values of LFD mean that with the increase of radius, the spectral cloud reflectance decreases (due to increase of absorption) as one might expect. Except for the case of thin clouds $(\mathrm{COT}=1)$, the values of $S\left(z_{k}, \lambda\right)$ are higher for larger wavelengths at the cloud top. It means that the channels with larger wavelengths have more sensitivity to the effective radius close to the cloud top. The maximum of the sensitivity is located at some distance from the cloud top. The results for effective radius equal to 6 and 18 micrometers are given in Fig. 2. At the wavelength $3.7 \mu \mathrm{m}$, the radiance is more sensitive to the size of particles in the case of smaller effective radius (at least in the upper portion of the cloud). It is the other way around for other wavelengths studied. The sensitivity of radiance to the effective radius increases with the VZA (see Fig. 3). Therefore, it is of importance to use slant observation geometry. Generally, the observation of cloud under different angles increases the information content of corresponding observations (Platnick, 2000).

\section{The solution of the inverse problem}

\subsection{Theory}

Equation (17) can be directly used for the solution of the inverse problem. E.g. it follows from Eq. (17) under assumption that $p_{a}(z)$ does not depend on $z$ (i.e. assuming that the relative variation of the effective radius profile does not depend on the height):

$p_{a}=y(\lambda)\left[\int_{z_{1}}^{z_{2}} \tilde{K}_{a}(z, \lambda) \mathrm{d} z\right]^{-1}$,

where $p_{a} \equiv \delta a_{\mathrm{ef}} / a_{\mathrm{ef}}$.

It means that the LFD investigated in the previous section can be directly used to find the variation

$p_{a}=\left(\hat{a}_{\mathrm{ef}}-a_{\mathrm{ef}}\right) / a_{\mathrm{ef}}$,

and, therefore, the value of the retrieved grain size $\hat{a}_{\text {ef }}$ can be easily found for a given value of

$y(\lambda)=\delta R(\lambda) / R\left(\lambda, a_{\mathrm{ef}}\right)=\left(R_{\mathrm{mes}}(\lambda)-R\left(\lambda, a_{\mathrm{ef}}\right)\right) / R\left(\lambda, a_{\mathrm{ef}}\right)$.

Here $R\left(\lambda, a_{\mathrm{ef}}\right)$ is the reflectance of a cloud for an assumed cloud droplet effective radius $a_{\text {eff }}$. The derived value of the effective radius (namely, $\hat{a}_{\mathrm{ef}}$ ) can be used instead of $a_{\mathrm{ef}}$ in equations given above to perform an iteration process. Clearly, due to the cloud vertical inhomogeneity, the derived value $\hat{a}_{\text {ef }}$ depends on the wavelength used in the measurements.

In real cloud layers the values of $p_{a}$ and $a_{\mathrm{ef}}$ depend on the vertical coordinate. Let us consider this more complex case now and assume that the profile of the effective radius can be presented in the following form:

$a_{\mathrm{ef}}(z)=\sum_{j=0}^{J} \alpha_{j} z^{j} ;$

in principle other parameterizations of profiles can be used as well. But the analysis of respective experimental measurements (Miles at al., 2000) show that the expansion (25) can indeed be used to explain most of profiles occurring at least in warm shallow clouds. The value of $J$ is usually not larger than 2. So quadratic, linear, and constant profiles can be used for the interpretation of respective measurements.

In the following discussion we restrict ourselves to the linear approximation of effective radius profiles $a_{\mathrm{ef}}(z)$. In particular, we set $J=1$ in Eq. (25) and rewrite it in the following equivalent form:

$a_{\mathrm{ef}}(z)=a_{t} \varphi(z)+a_{b}(1-\varphi(z))$,

where $\varphi(z)=\left(z_{1}-z\right)\left(z_{1}-z_{2}\right)^{-1}, a_{t}$ and $a_{b}$ are the effective radii at the cloud top and bottom, respectively.

The substitution of Eq. (26) in Eq. (17) gives:

$y(\lambda)=c_{1}(\lambda) \delta a_{t}+c_{2}(\lambda) \delta a_{b}$, 


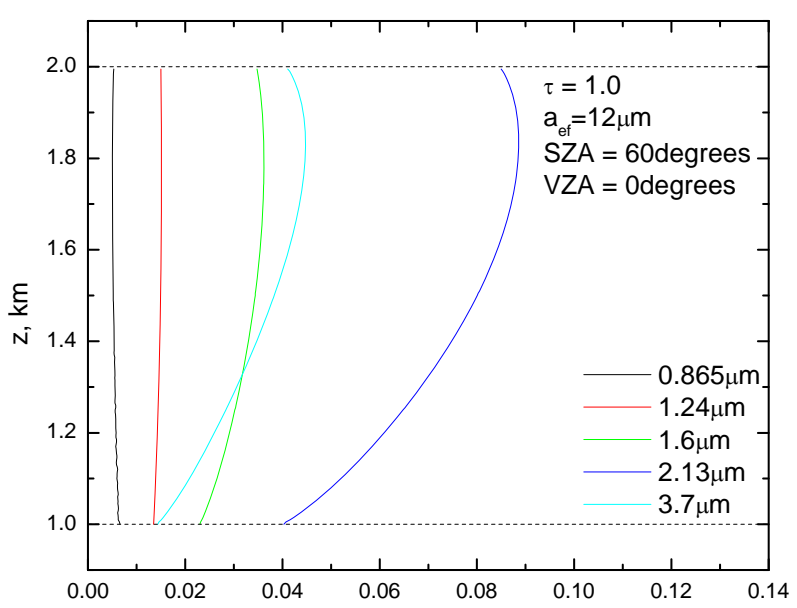

$|\mathrm{S}|, \mathrm{km}^{-1}$

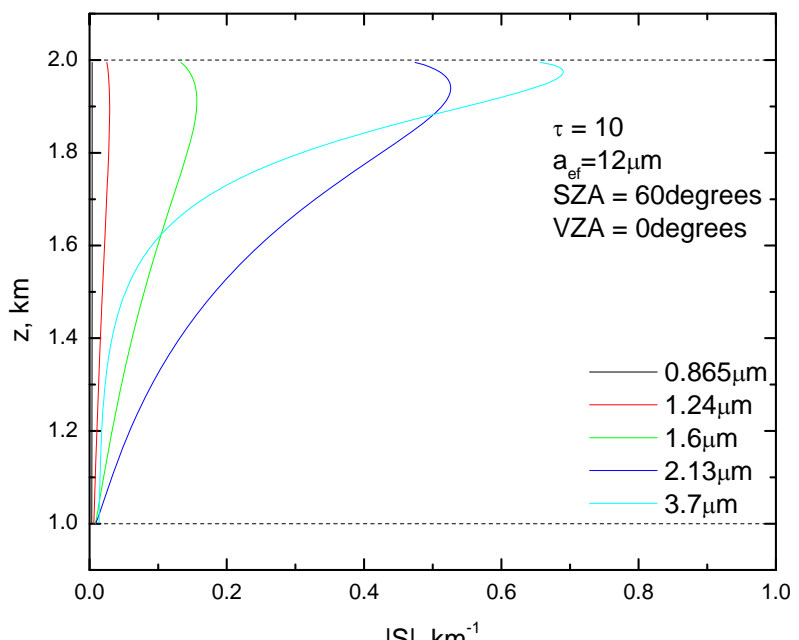

$|\mathrm{S}|, \mathrm{km}^{-1}$

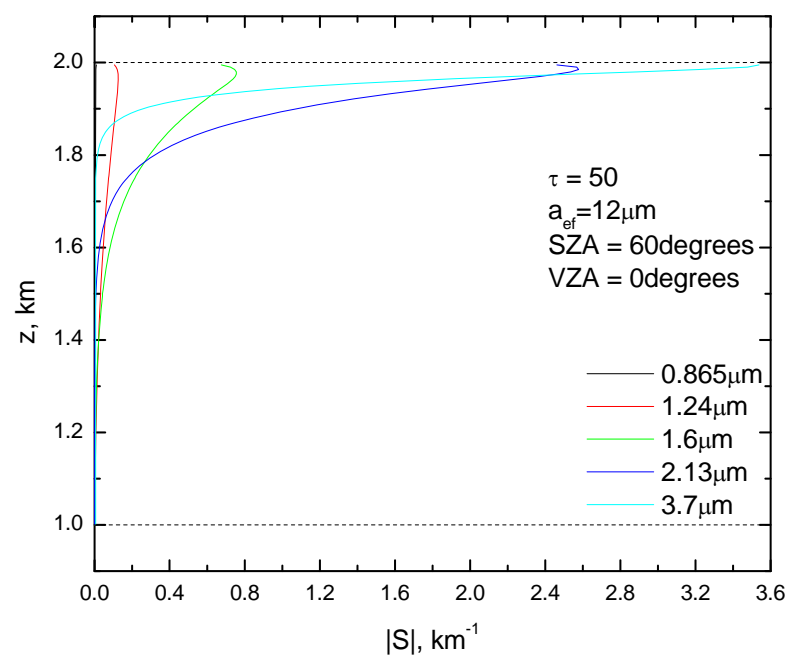

(a)

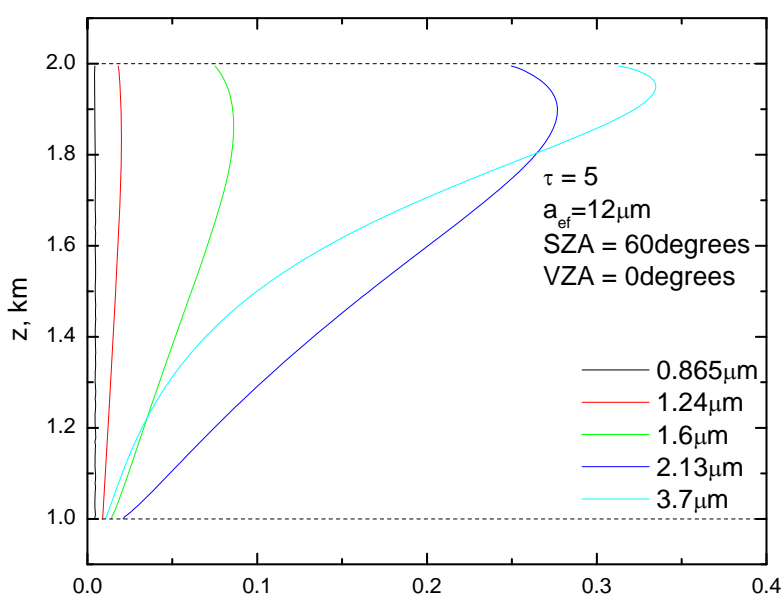

$|\mathrm{S}|, \mathrm{km}^{-1}$

(b)

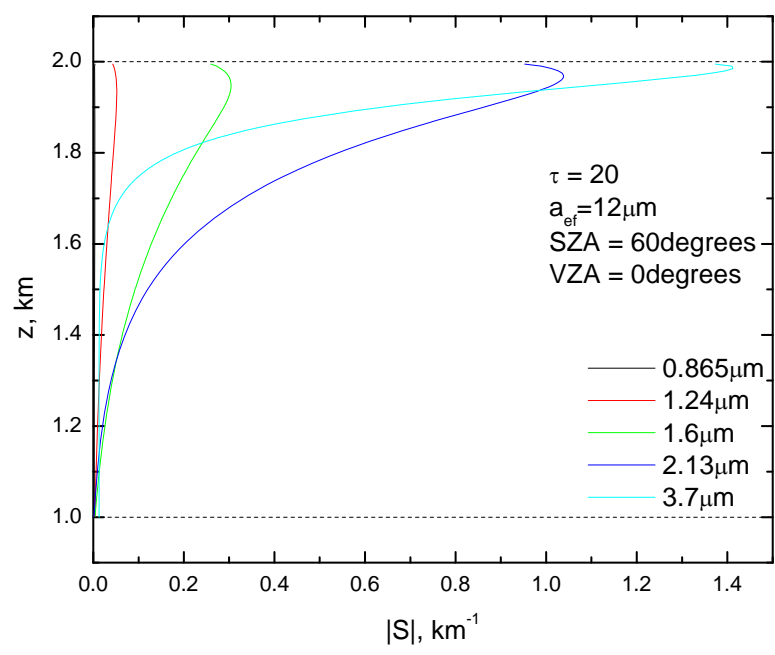

(d)

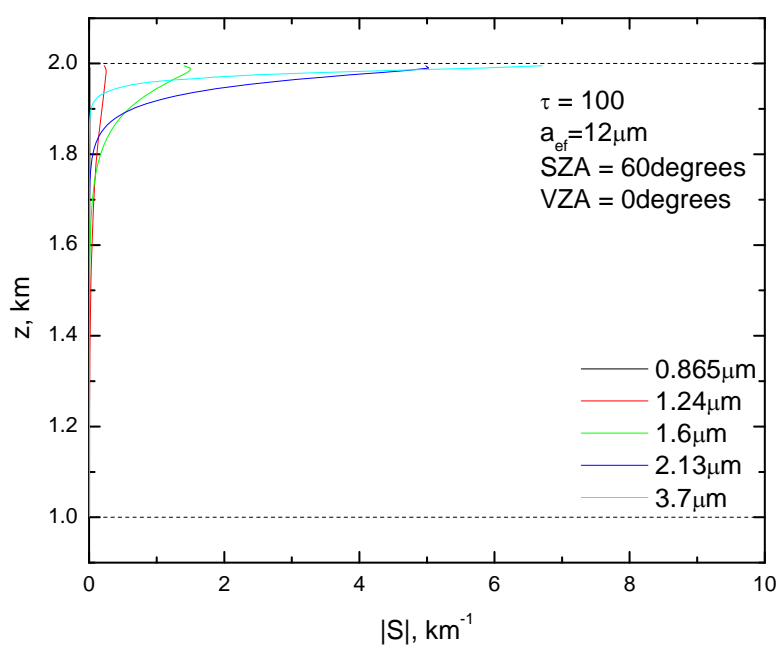

(f)

Fig. 1. The vertical profiles of the absolute value of $S\left(z_{k}\right)$ for various wavelengths at COT $=1(\mathbf{a}), 5(\mathbf{b}), 10(\mathbf{c}), 20(\mathbf{d}), 50(\mathbf{e}), 100(\mathbf{f})$ at $a_{\mathrm{ef}}=12 \mu \mathrm{m}(\mathrm{VZA}=0$ degrees, SZA $=60$ degrees). The cloud is positioned between 1 and $2 \mathrm{~km}$. Rayleigh scattering and thermal emission are taken into account. Aerosol scattering and absorption are ignored. 


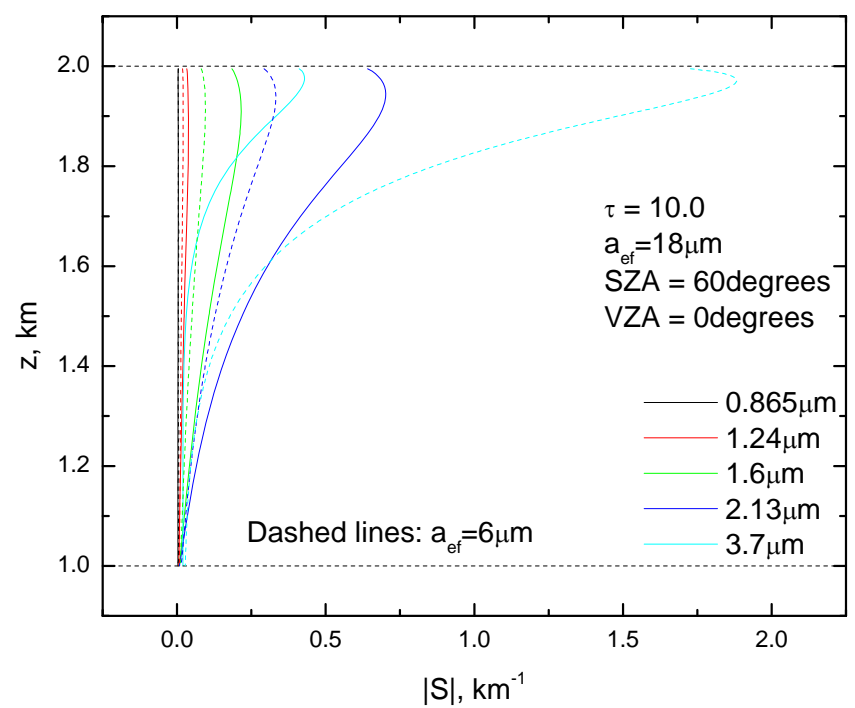

Fig. 2. The same as in Fig. 1c except at $E R=6$ and $18 \mu \mathrm{m}$.

where

$$
\begin{aligned}
& c_{1}(\lambda)=\int_{z_{1}}^{z_{2}} \frac{\tilde{K}_{a}(z, \lambda) \varphi(z) \mathrm{d} z}{a_{\mathrm{ef}}(z)}, \\
& c_{2}(\lambda)=\int_{z_{1}}^{z_{2}} \frac{\tilde{K}_{a}(z, \lambda) \mathrm{d} z}{a_{\mathrm{ef}}(z)}-c_{1}(\lambda) .
\end{aligned}
$$

The function $\tilde{K}_{a}(z, \lambda)$ can be calculated using SCIATRAN as discussed above for an assumed a priori profiles $a_{\mathrm{ef}}(z), N(z)$. This enables also the calculation of coefficients $c_{1}(\lambda)$ and $c_{2}(\lambda)$.

The linear model as presented in Eq. (27) is derived under assumption that the variation of cloud reflectance is caused by the variation of the effective radius at cloud top and bottom only. However, the cloud optical thickness is usually obtained with limited accuracy. Therefore, to account for the possible variation of cloud optical thickness, we rewrite liner model given by Eq. (27) as follows:

$y(\lambda)=c_{1}(\lambda) \delta a_{t}+c_{2}(\lambda) \delta a_{b}+c_{3}(\lambda) \delta \tau_{0}$,

where the last term on the right-hand side of this equation describes the variation of optical thickness and $c_{3}(\lambda)=\frac{\partial \ln R}{\partial \tau_{0}}$. The last term in Eq. (29) enables the improvement of the initial estimation of COT.

Let us rewrite Eq. (29) in the vector-matrix form:

$\boldsymbol{y}=\hat{\mathrm{K}}\left(\boldsymbol{x}-\boldsymbol{x}_{0}\right)$.

The components of the vector $\boldsymbol{y}$ are relative changes of the reflectances at each measured wavelength $\lambda$ (as compared to the spectral cloud reflectance for a priori profile), $\boldsymbol{x}=\left\{a_{t}, a_{b}, \tau_{0}\right\}^{T}$ is the vector parameter to be found and $\boldsymbol{x}_{0}=\left\{\bar{a}_{t}, \bar{a}_{b}, \bar{\tau}_{0}\right\}^{T}$ is a priori state vector, which we will derive

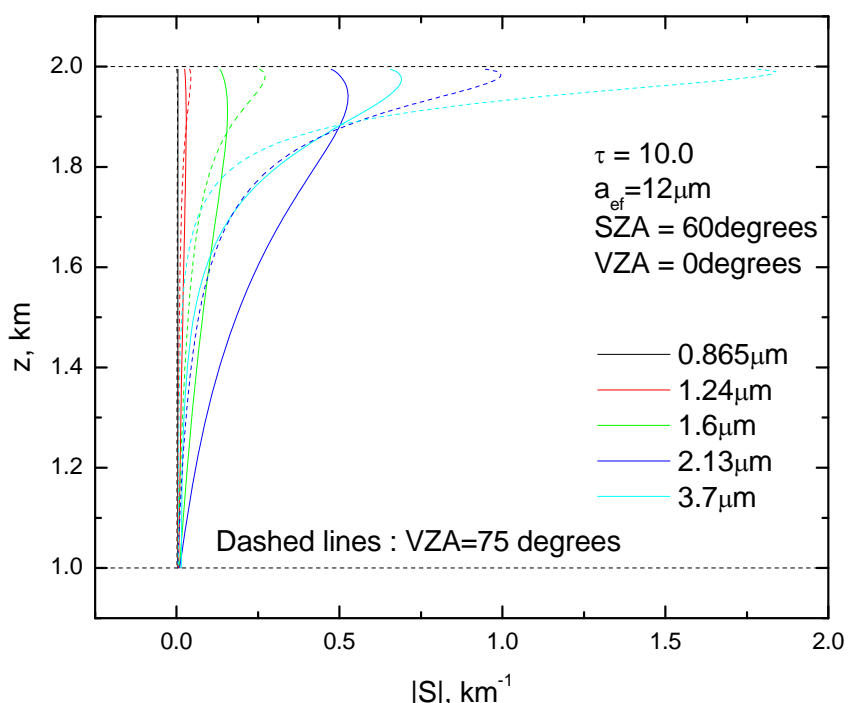

Fig. 3. The same as in Fig. 1c except the results at VZA equal to 75 degrees are also given (dashed lines).

applying the standard retrieval procedure for a homogeneous cloud layer (Kokhanovsky, 2006). This means that we assume that $\bar{a}_{t}=\bar{a}_{b}$ for the first guess.

Equation (30) is a typical example of the linearized inverse problem. This linear ill-posed problem is solved in the least squares sense employing the generalized Tikhonov regularization, i.e. minimizing the following quadratic form:

$\left\|\boldsymbol{y}-\hat{\mathrm{K}}\left(\boldsymbol{x}-\boldsymbol{x}_{0}\right)\right\|_{\mathbf{S}_{y}^{-1}}^{2}+\left\|\boldsymbol{x}-\boldsymbol{x}_{0}\right\|_{\mathbf{Q}}^{2}=\min$.

Here $\mathbf{S}_{y}^{-1}$ is the inverse error covariance matrix of the measurement vector $\boldsymbol{y}$ and $\mathbf{Q}$ is the constraint matrix for the state vector $\boldsymbol{x}$. Following Rodgers (2008), the state vector constraint matrix is represented by the inverse covariance matrix, i.e. $\mathbf{Q}=\mathbf{S}_{a}^{-1}$. The solution of Eq. (31) is obtained in the following analytical form (Rodgers, 2008):

$\boldsymbol{x}=\boldsymbol{x}_{0}+\left(\hat{\mathrm{K}}^{T} \mathbf{S}_{y}^{-1} \hat{\mathrm{K}}+\mathbf{S}_{a}^{-1}\right)^{-1} \hat{\mathrm{K}}^{T} \mathbf{S}_{y}^{-1} \boldsymbol{y}$

All matrices, $\boldsymbol{x}_{0}$ and $\boldsymbol{y}$ are known functions (for an assumed a priori profile $a_{\mathrm{ef}}(z)$ and $\left.\tau_{0}\right)$. To account for the non-linearity of the inverse problem, the Gauss-Newton iterative approach (Rodgers, 2008) is used. At $i+1$ iterative step, this approach results in the following solution:

$\boldsymbol{x}_{i+1}=\boldsymbol{x}_{i}+\left(\hat{\mathrm{K}}_{i}^{T} \mathbf{S}_{y}^{-1} \hat{\mathrm{K}}_{i}+\mathbf{S}_{a}^{-1}\right)^{-1} \hat{\mathrm{K}}_{i}^{T} \mathbf{S}_{y}^{-1} \boldsymbol{y}_{i}$,

where the subscript " $i$ " denotes that corresponding quantities are calculated using the vector-parameter $\boldsymbol{x}_{i}$. The iterative process is stopped if the maximum difference between the components of the state vector at two subsequent iterative steps does not exceed a given criterion. Clearly, for initial 


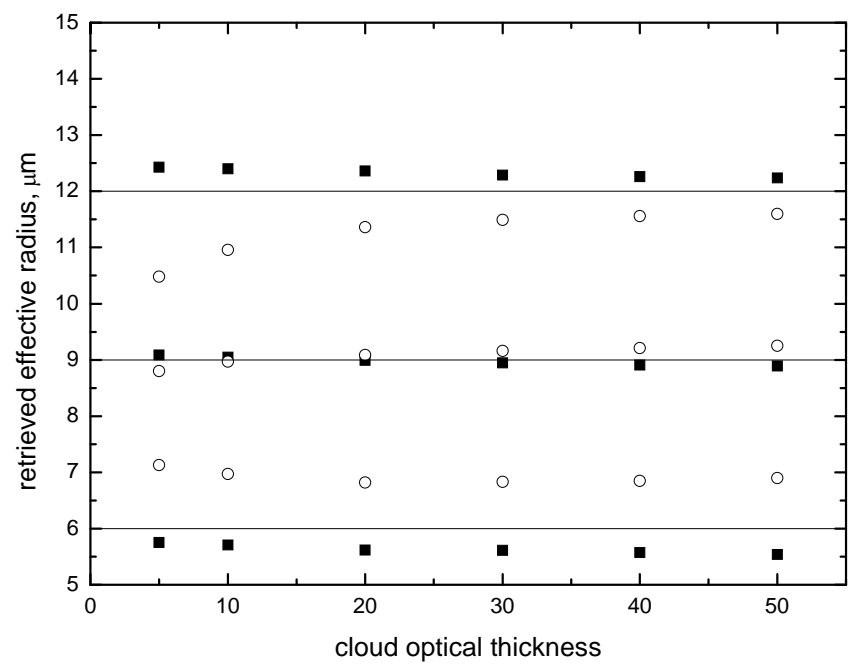

Fig. 4a. The dependence of the retrieved effective radius on different levels in the cloud on the value of the cloud optical thickness. The true linear profile is $12-9-6 \mu \mathrm{m}$. The first number denotes the values of $a_{\mathrm{ef}}$ at the cloud top, the second number corresponds to the middle of the cloud and the last number is the droplet effective radius at the cloud bottom. The SZA is equal to 60 degrees and the observation is at the nadir direction for the black underlying surface. Squares correspond to the case when the cloud is positioned at 1$3 \mathrm{~km}$ in the retrieval process (not $1-2 \mathrm{~km}$ as in the forward synthetic run), and circles correspond to the case of the cloud positioned in the range of heights $0.5-1 \mathrm{~km}$ in the retrieval process.

profiles $a_{\mathrm{ef}}(z)$ and $\tau_{0}$ close to the sought profile, fast convergence can be achieved. This is illustrated in the next section for the case of linear profiles.

We use the asymptotic radiative transfer theory for the first guess estimates of COT and the effective radius, i.e. it is assumed that $\bar{a}_{t}=\bar{a}_{b}=a_{\mathrm{ef}, s}$, where $a_{\mathrm{ef}, s}$ is the effective radius derived using the inversion of the asymptotic radiative transfer equations as applied to a vertically homogeneous cloud layer (Kokhanovsky, et al., 2003; Kokhanovsky, 2006). The retrieved COT and effective radius are the values derived for an assumed homogeneous cloud of the geometrical thickness equal to $500 \mathrm{~m}$. The influence of profile of effective radius on the retrieved value of COT is weak. The retrieved profile of effective radius is improved using iterations until the convergence is reached. This completes the description of the retrieval algorithm.

\subsection{Retrievals}

\subsubsection{Synthetic retrievals}

We show the capability of the newly developed code for the retrieval of the Profile of the Effective Radius using the Optimal estiMation Technique (PEROMT) in shallow water clouds using synthetic top-of-atmosphere reflectances as derived from SCIATRAN simulations. In particular, the values

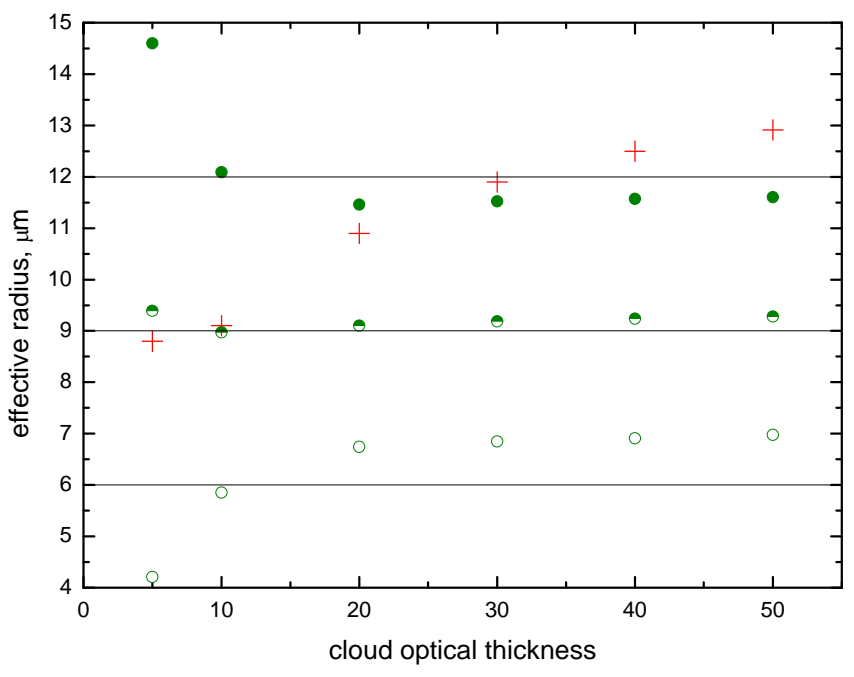

Fig. 4b. The same as in Fig. 4a except it was assumed in the retrieval process that the cloud positioned in the range of heights $0.5-$ $1 \mathrm{~km}$ and the underlying surface albedo is 0.1 (not 0.0 as in the forward model). The crosses show the results of retrievals based on the semi-analytical cloud retrieval algorithm for a vertically homogeneous cloud.

of the top-of-atmosphere cloud reflectance were calculated at the wavelengths $0.865,1.22,1.6,2.2 \mu \mathrm{m}$ for the vertically inhomogeneous clouds with $a_{t}=12 \mu \mathrm{m}, a_{b}=6 \mu \mathrm{m}$ over black underlying surface. We did not use the channel at $3.7 \mu \mathrm{m}$ to be less sensitive to a priori unknown cloud position in atmosphere. It was assumed that SZA is equal to 60 degrees and the observation is in the nadir direction. The molecular scattering was accounted for but the aerosol contribution was set to zero in the forward and inverse models. In the retrieval process the error covariance matrix $\mathbf{S}_{y}$ and a priori covariance matrix $\mathbf{S}_{a}$ have been used as diagonal matrices. The square of diagonal elements of $\mathbf{S}_{y}$ corresponds to the relative measurement error of $0.1 \%$. The a priori uncertainties for $a_{t}$ and $a_{b}$ elements of the state vector were set to 10 and $1 \mu \mathrm{m}$, respectively, and to $0.1 \tau_{0}$ for the optical thickness.

The results of the retrievals of parameters $a_{b}, a_{t}$ (and also $a_{\mathrm{m}}$ (at the middle of the cloud)) as functions of COT are illustrated in Fig. 4a, assuming that the surface albedo is the same as in the forward simulation but the cloud position is wrong (1-3 and $0.5-1 \mathrm{~km}$ and not $1-2 \mathrm{~km}$ as in the forward simulation). We found that the errors in the vertical profile of effective radius are below $1 \%$, if the cloud position is exactly known in advance.

The cloud top height error of $+1 \mathrm{~km}$ in Fig. $4 \mathrm{a}$ is close to the upper cloud top height retrieval error, depending on the instrument used (thermal infrared, oxygen A-band, $\mathrm{CO}_{2}$ band cloud top height retrieval techniques). We conclude that neither COT not the error in the cloud top height of $1 \mathrm{~km}$ influences the retrieval results considerably. In all cases the profile with particles increasing to the cloud top (as it should 


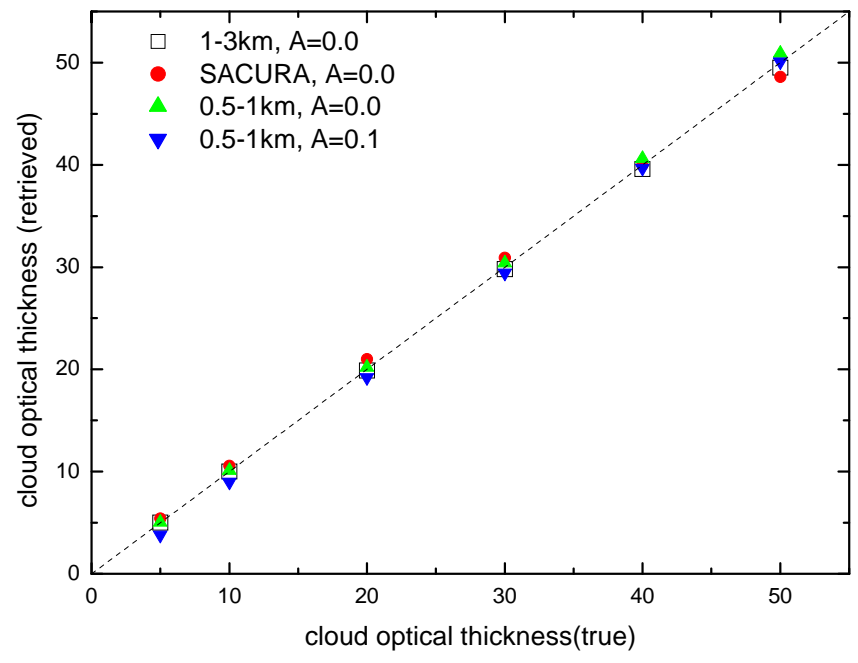

Fig. 5. The correlation plot of the retrieved versus true COT. Circles give the results of the retrieval based on the semi-analytical cloud retrieval algorithm for a homogeneous cloud (the first guess solution for the optimal estimation approach). Squares correspond to the case of a cloud positioned between $1-3 \mathrm{~km}$ and triangles correspond to the cloud positioned between $0.5-1 \mathrm{~km}$ ( for the assumed underlying surface albedo 0.0 and 0.1 ) in the retrieval process. The true underlying albedo was equal to $0.0, \mathrm{SZA}=60^{\circ}, \mathrm{VZA}=0^{\circ}$.

be) is found. The error of the retrieved effective radius is smaller than $0.5 \mu \mathrm{m}$ for the assumed cloud positioned between $1-3 \mathrm{~km}$ for all studied COTs, and it is below $1 \mu \mathrm{m}$ at all levels in the cloud for the assumed cloud positioned between $0.5-1 \mathrm{~km}$ and $\mathrm{COT} \geq 10$. For thinner clouds (positioned in the range $0.5-1 \mathrm{~km}$ in the retrieval process), the errors are larger but they are below $1.5 \mu \mathrm{m}$, if COT $\geq 5$ (see Fig. 4a). The information on the underlying surface albedo is crucial for the case of thin clouds. This is illustrated in Fig. $4 \mathrm{~b}$, where we show the dependence of the retrieved effective radius on three levels in the cloud for the case of the assumed surface albedo 0.1 (true surface albedo is equal to 0.0 ) and the cloud positioned in the range $0.5-1 \mathrm{~km}$. This is the worse scenario (see Fig. 4a) because not only cloud top height but also cloud bottom height used in the retrieval process are biased (from the position 1-2 km used in forward simulations). The errors increase considerably for the thin clouds $(\mathrm{COT}=5)$. It follows from Fig. $4 \mathrm{~b}$ that the bias of 0.1 in the assumed surface albedo does not influence results for thicker clouds. Crosses in Fig. 4b correspond to the case of the effective radius using the homogeneous cloud model. Just one value is retrieved and the results derived are closer to the true effective radius at some level in the cloud close to the top. The actual level on which effective radius is retrieved (if the homogeneous cloud model is used) depends not only on the wavelength used in the retrieval (here the wavelength at $1.6 \mu \mathrm{m}$ was used) but also on the COT (see Fig. 4b) and vertical profile of effective radius (not shown here). Therefore, the technique as proposed here has an advantage as far as one

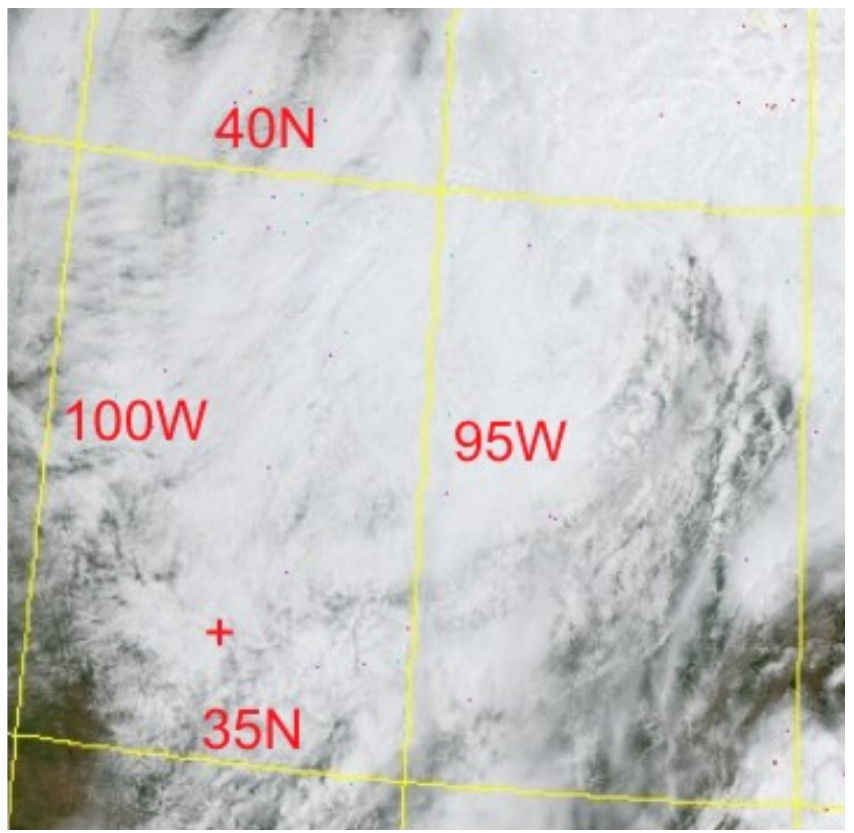

Fig. 6. The MODIS/TERRA browse image of the studied cloud scene (31 May 2001, 16:55 UTC). The cross signifies the position of the ARM site $\left(36.61^{\circ} \mathrm{N}, 97.49^{\circ} \mathrm{W}\right)$.

is interested in the vertical distribution of effective radius of water droplets in clouds. Clearly, the bias in the retrieved effective radius inherent to the homogeneous cloud model will also lead to a bias in the retrieved liquid water path (LWP), which is usually found as LWP $=2 \tau_{0} \rho a_{\mathrm{ef}} / 3$, where $\rho$ is the density of water. The vertical inhomogeneity of cloud does not lead to considerable biases in the retrieved cloud optical thickness $\tau_{0}$ (see Fig. 5). Therefore, we conclude that the retrieved LWP is overestimated for the case considered here (the effective radius increases towards the cloud top). This is due to the fact that the value of $a_{\text {ef }}$ retrieved using the homogeneous cloud model is close to that at the top and, therefore, overestimated.

\subsubsection{Retrievals using MODIS data over land}

Chang and Li (2003) applied their look-up table method for the cloud system located over ARM SGP site $\left(35.269^{\circ} \mathrm{N}\right.$, $96.74^{\circ} \mathrm{W}$ ) using MODIS Terra data for 31 May 2001 (see Fig. 6). The cloud was located between 0.8 and $1.5 \mathrm{~km}$ as measured by the ground-based instrumentation at ARM. The cloud top temperature was $283.1 \mathrm{~K}$ (Chang and $\mathrm{Li}, 2003$ ). Therefore, we are sure that crystals do not occur in this cloud.

Interestingly, different profiles were obtained by Chang and $\mathrm{Li} \mathrm{(2003)}$ using the combination of channels 3.7/2.1 and $3.7 / 1.6 \mu \mathrm{m}$. In particular, their retrievals using the combination 3.7/2.1 $\mu \mathrm{m}$ gave almost homogeneous cloud $(\mathrm{ER}=10.5 \mu \mathrm{m}$ (top), $\mathrm{ER}=9 \mu \mathrm{m}$ (bottom)). The retrievals using $3.7 / 1.6 \mu \mathrm{m}$ gave the profile $\mathrm{ER}=10.5 \mu \mathrm{m}$ (top), 


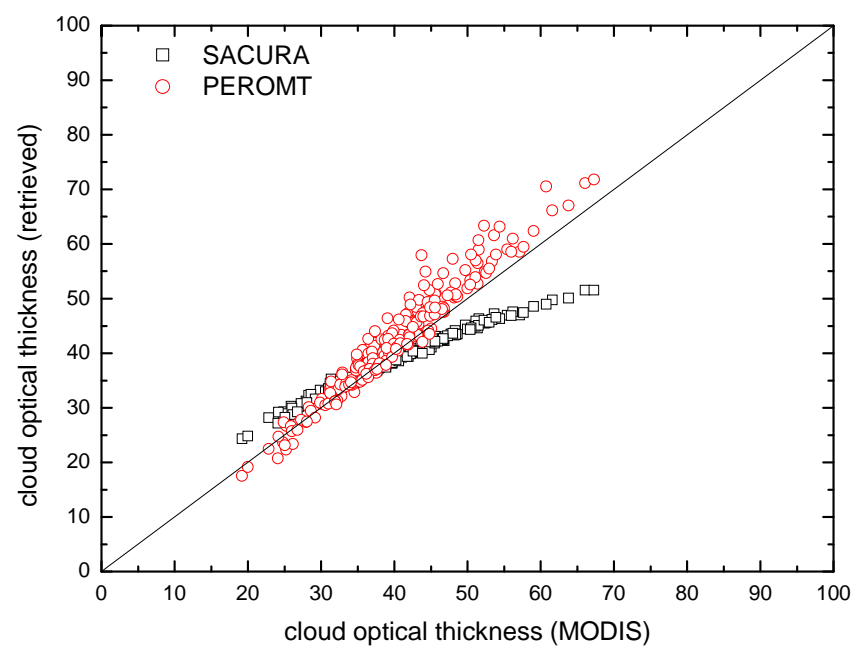

Fig. 7a. The correlation between COT derived using MODIS algorithm and SACURA, PEROMT retrievals.

$\mathrm{ER}=5 \mu \mathrm{m}$ (bottom). The differences are significant. We suggest that the combination 3.7/1.6 $\mu \mathrm{m}$ provides better estimation of profile because of larger separation of wavelengths and deeper sampling into the cloud. Our retrievals for the same cloud system centered at the ARM SGP location (averaged over a region $97.45 \pm 0.08^{\circ} \mathrm{W}$ and $36.59 \pm 0.06^{\circ} \mathrm{N}$, similar to the area used for averaging by Chang and $\mathrm{Li}$ (2003)), gave: $9.94 \pm 1.1 \mu \mathrm{m}$ (top), $7.0 \pm$ $0.6 \mu \mathrm{m}$ (middle), $4.0 \pm 0.04 \mu \mathrm{m}$ (bottom) - close to the estimates of Chang and $\mathrm{Li}$ (2003) for the pair 3.7/1.6 $\mu \mathrm{m}$. The derived COT $=44.66 \pm 12.5$ was also close to that reported by Chang and $\mathrm{Li}(2003)(38.7 \pm 4.2)$ and also to that reported by MODIS $(42.29 \pm 10.9)$ and the groundbased measurements (37.4). The COT retrieved by SACURA (Kokhanovsky et al., 2003) was $39.82 \pm 5.8$, which was close to the ground based measurements of COT.

This comparison shows that both methods (PEROMT and Cheng and $\mathrm{Li}$ (2003) technique) produce similar results. In particular, the effective radius increasing towards cloud top height is found in both retrievals. This is characteristic for the cases with no drizzle. Actually, no drizzle was reported at ARM SGP site at the time of MODIS measurements. However, the technique developed by us is more flexible and enables the extension taking into account other profiles of effective radii and channels without substantial modification of the algorithm. The previously developed method (Cheng and $\mathrm{Li}, 2003$ ) will require the production of new look-up tables if other cases or profiles are of interest. As a matter of fact, this paper gives the first application of optimal estimation approach in the framework of exact radiative transfer calculations with respect to the cloud droplet profile determination.

The results of retrievals for the ARM SGP site are presented in more detail in Fig. 7. We assumed that the surface

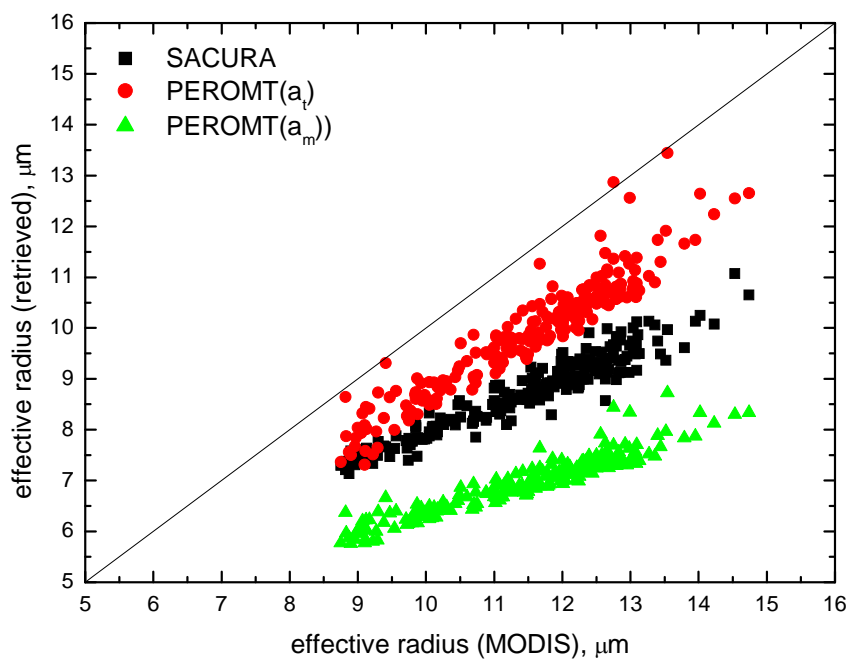

Fig. 7b. The same as in Fig. 7a except for the effective radius.

albedo is 0.5 in the retrieval process. We found that the assumption on the surface albedo almost does not influence the results of retrievals (cloud optical thickness, vertical profile of effective radius). This is due to the high value of COT for the cloud under study.

It follows from Fig. 7a that the PEROMT algorithm retrieves COTs, which are comparable to the MODIS result. The result of SACURA, which is used as a first guess, is biased because SACURA is based on the approximation of the radiative transfer equation and small errors of the approximation amplify for thicker clouds. In Fig. $7 \mathrm{~b}$ we show the correlation plots of the SACURA and PEROMT-derived effective radii with that of MODIS. The correlation coefficients are high. However, generally, the MODIS results are higher as compared to our retrievals. This can be related to the fact that the half-width of the size distribution and also the refractive index of water used in MODIS retrieval does not exactly coincide with the assumptions used in our retrieval. This is especially important in the geometries corresponding to rainbow and glory scattering. In particular, the average SZA, VZA, and relative azimuth angle for the case studied by us were $24.4,53.5$, and 95.3 degrees, respectively. This gives the scattering angle of 125 degrees, which is close to the position of the secondary rainbow (129 degrees according to the geometrical optics calculations).

One concludes from Fig. $7 \mathrm{~b}$ that the assumption of a homogeneous cloud (as used in SACURA) gives the effective radius at some level close to the cloud top. The retrievals using PEROMT give the improvement of the standard cloud retrieval techniques providing the values of effective radius throughout the cloud. The derived profiles of effective radius, if used in the radiative transfer simulations, give the spectral reflectance coefficients, which are close to the measured ones for all selected channels (the average rms is 0.017). 

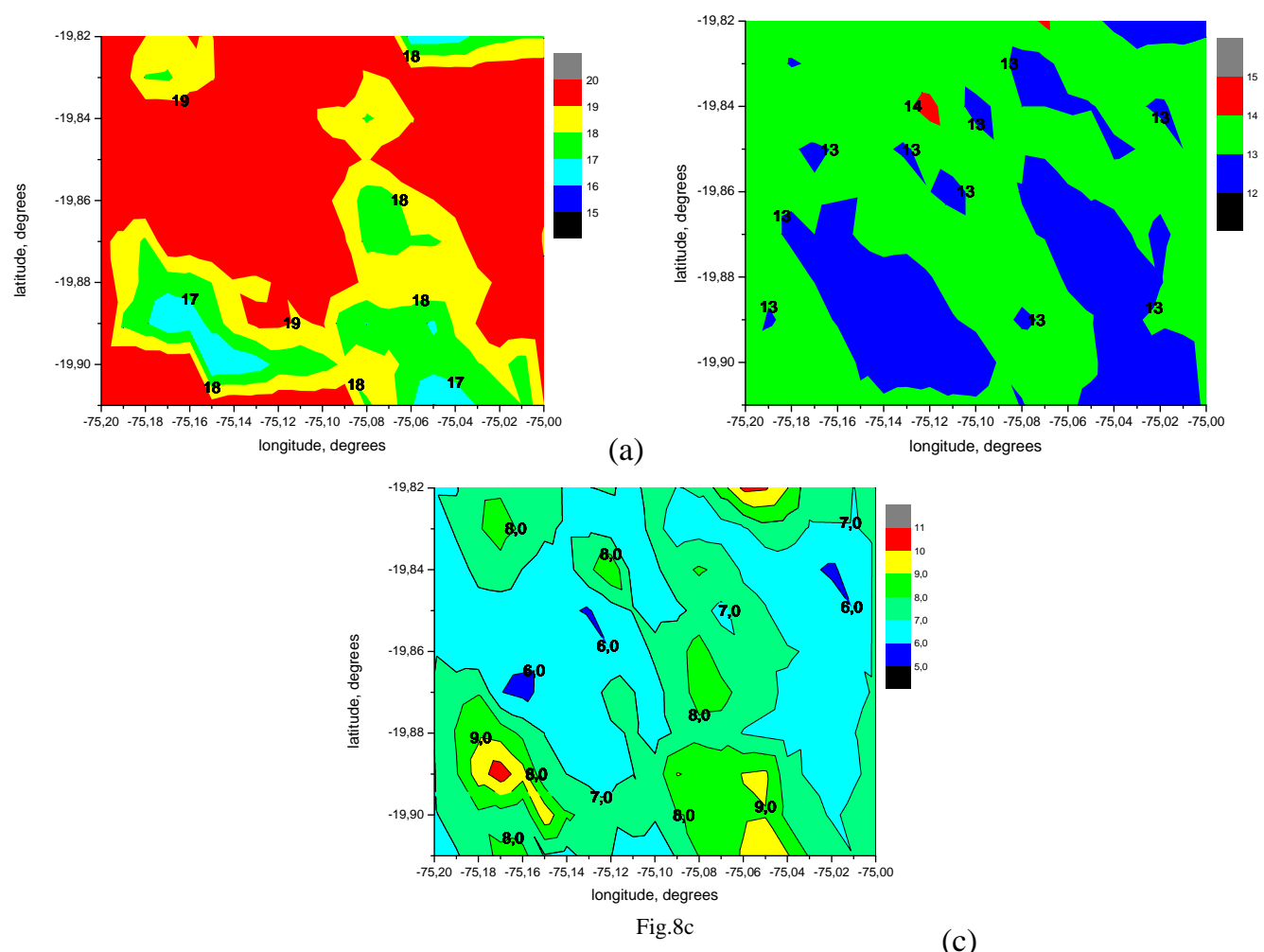

(c)

Fig. 8. The map of the effective radius retrieved using PEROMT at the cloud top (a), middle (b), and bottom (c).

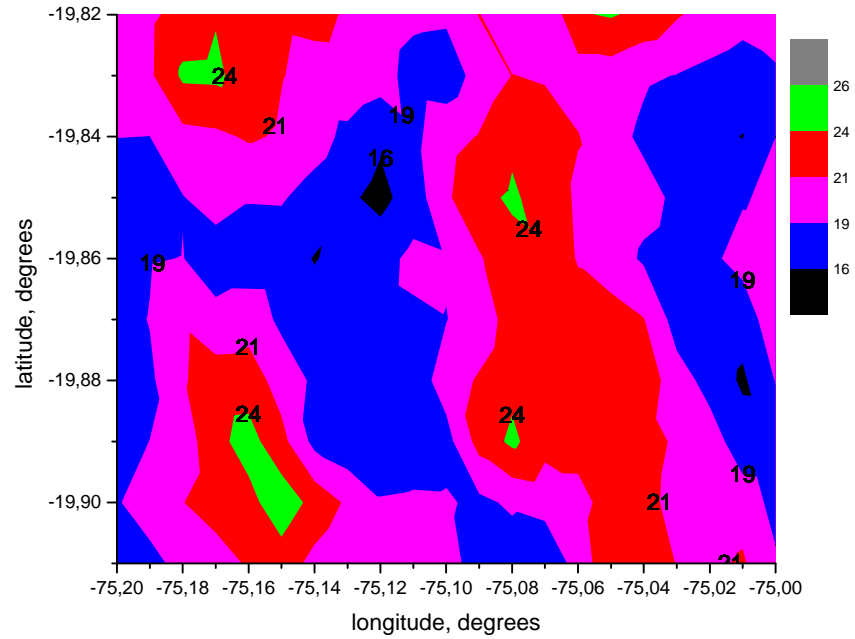

Fig. 9. The map of COT retrieved using PEROMT.

\subsubsection{Retrievals using MODIS data over ocean}

The results of retrievals using a MODIS scene over ocean (west of Chile, the center of the scene is located at $75.09^{\circ} \mathrm{W}$, $19.87^{\circ} \mathrm{S}$ ) are presented in Fig. 8, where the retrieved droplet effective radius spatial distributions at the top (a), middle (b) and bottom (c) of cloud are given. The retrieved cloud optical

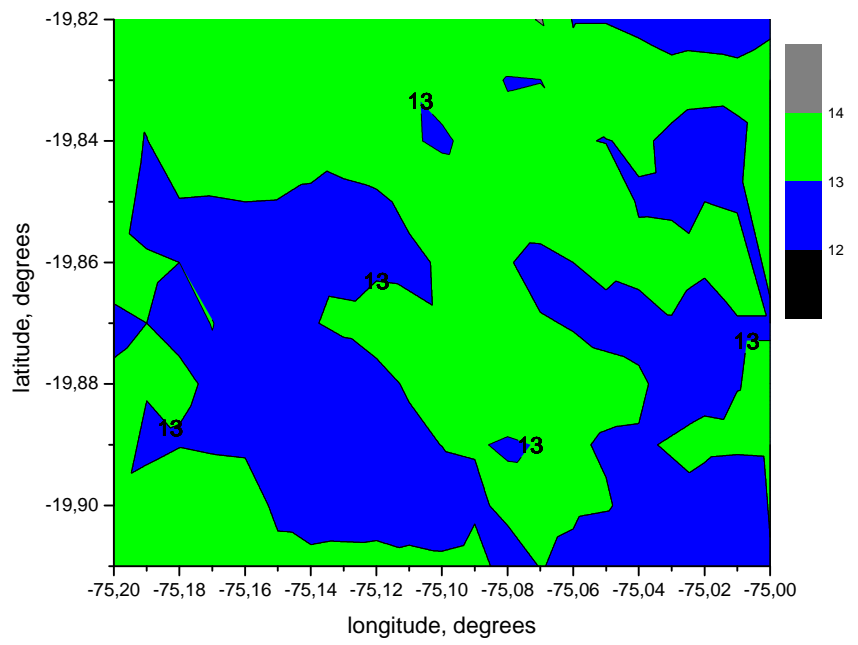

Fig. 10. The spatial distribution of $a_{\mathrm{ef}}$ as derived using MODIS operational algorithm.

thickness is presented in Fig. 9. The average COT for the scene was $20.1 \pm 2.3(25.5 \pm 3$ as derived by the MODIS operational algorithm). It was assumed that the surface albedo is equal to zero in the retrieval process although deviations from this value do not change results due to large thickness of the cloud. The cloud was positioned between 2.8 
and $3.3 \mathrm{~km}$, where the upper cloud boundary was estimated from corresponding MODIS retrievals of cloud top pressure. The derived average values of $a_{\mathrm{ef}}$ were $18.8 \pm 1.2 \mu \mathrm{m}$ (top), $13.1 \pm 0.4 \mu \mathrm{m}$ (middle), and $7.4 \pm 1.0 \mu \mathrm{m}$ (bottom). The droplets are larger as compared to the case of clouds over land discussed in the previous section. The droplet size decreases towards the cloud bottom, which could signify the absence of precipitation although droplets are quite large at the cloud top. The spatial distribution of $a_{\mathrm{ef}}$ as derived from MODIS is given in Fig. 10. It follows that $a_{\mathrm{ef}}=13.1 \pm 0.4 \mu \mathrm{m}$, which is close to the results derived using SACURA $\left(a_{\mathrm{ef}}=13 \pm 0.5 \mu \mathrm{m}, \tau=23.9 \pm 2.7\right)$ and also to the spatial distribution of $a_{\mathrm{ef}}$ in the middle of the cloud (compare Figs. 8b and 10).

\section{Conclusions}

A technique to retrieve cloud droplet vertlical profiles has been developed. Theoretically, the method can be applied to the arbitrary profiles of the droplet effective radius. However, the authors restrict this work to the linear profiles. The method was applied to the synthetic data and it was shown that the technique is not very sensitive neither to the uncertainty to the underlying surface albedo (for optically thick clouds) nor to the uncertainty in the cloud position. We believe that the method is best suited for clouds with COTs above approximately 10 . The technique was also applied to the satellite data, where we found that the conventional cloud retrieval technique (for a homogeneous cloud) produces the radii close to those in the upper portion of the cloud (or even close to those at the middle of the cloud as in the analyzed case of a maritime shallow cloud). Our technique is not based on LUTs. Therefore, it can be used without large alterations for many types of satellite instruments. Clearly, adding the multi-angular and polarization measurements in the optimal estimation framework provided here can enhance the accuracy of retrievals. The next important step in the development of the method is its validation using in-cloud measurements of profiles $a_{\mathrm{ef}}(z)$. The method has several limitations. In particular, it is applicable mostly to the low-level horizontally homogeneous marine clouds with warm microphysics.

\section{Appendix A}

\section{The relative variation of the droplet number concentration}

The aim of this Appendix is to provide the derivation of the relationship between the relative variation of the droplet number concentration $p_{N}$ and the variation of the droplet effective radius $p_{a}$ under assumption that COT at the wavelength $\lambda_{0}$ is exactly known.
The variation of COT can be presented in the following form:

$\delta \tau\left(\lambda_{0}\right)=\int_{z_{1}}^{z_{2}} \delta \kappa_{\mathrm{ext}}\left(z, \lambda_{0}\right) d z$.

Taking into account that $\delta \tau\left(\lambda_{0}\right)=0$ in our case and (see Sect. 2.2)

$$
\begin{aligned}
\kappa_{\mathrm{ext}}\left(z, \lambda_{0}\right)= & N(z)<C_{\mathrm{ext}}\left(z, \lambda_{0}\right)>, \\
\delta \kappa_{\mathrm{ext}}\left(z, \lambda_{0}\right)= & \delta N(z)<C_{\mathrm{ext}}\left(z, \lambda_{0}\right)> \\
& +N(z) \frac{\partial<C_{\mathrm{ext}}\left(z, \lambda_{0}\right)>}{\partial a_{\mathrm{ef}}(z)} \delta a_{\mathrm{ef}}(z),
\end{aligned}
$$

we derive from Eq. (A1):

$$
\begin{aligned}
\int_{z_{1}}^{z_{2}}[ & \delta N(z)<C_{\mathrm{ext}}\left(z, \lambda_{0}\right)> \\
& \left.+N(z) \frac{\partial<C_{\mathrm{ext}}\left(z, \lambda_{0}\right)>}{\partial a_{\mathrm{ef}}(z)} \delta a_{\mathrm{ef}}(z)\right] d z=0
\end{aligned}
$$

or assuming that $p_{N}$ does not depend on $z$ :

$p_{N}=-\frac{1}{\tau_{0}} \int_{z_{1}}^{z_{2}} \frac{\partial \kappa_{\mathrm{ext}}\left(z, \lambda_{0}\right)}{\partial \ln a_{\mathrm{ef}}(z)} p_{a}(z) d z$.

Acknowledgements. This work was supported by the DFG Project RESINC-2. The authors thank Anja Hünerbein for help with processing MODIS data.

Edited by: J. Cermak

\section{References}

Chang, F.-L. and Li, Z.: Estimating the vertical variation of cloud droplet effective radius using multispectral near - infrared satellite measurements, J. Geophys. Res., 107, 1-12, 2002.

Chang, F.-L. and Li, Z.: Estimating the vertical variation of cloud droplet effective radius: Algorithm modification and preliminary application, J. Geophys. Res., 108, 4763, doi:10.1029/2003JD003906, 2003.

Kokhanovsky, A. A., Rozanov, V. V., Zege, E. P., Bovensmann, H., and Burrows, J. P.: A semi-analytical cloud retrieval algorithm using backscattered radiation in 0.4-2.4 micrometers spectral range, J. Geophys. Res., 108, 4008, doi:10.1029/2001JD001543, 2003.

Kokhanovsky, A. A.: Cloud Optics, Berlin: Springer, 2006.

Miles, N. L., Verlinde, J., and Clothiaux, E. E.: Cloud droplet size distributions in low-level stratiform clouds, J. Atmos. Sci., 57, 295-311, 2000.

Nakajima, T. Y., Suzuki, K., and Stephens, G. L.: Droplet growth in warm water clouds observed by the A-Train. Part I: Sensitivity analysis of the MODIS-derived cloud droplet sizes, J. Atmos. Sci., 67, 1884-1896, 2010a. 
Nakajima, T. Y., Suzuki, K., and Stephens, G. L.: Droplet growth in warm water clouds observed by the A-Train. Part II: A multisensor view, J. Atmos. Sci., 67, 1897-1907, 2010 b.

Platnick, S.: Vertical photon transport in cloud remote sensing problems, J. Geophys. Res., 105, 22919-22935, 2000.

Rodgers, C.: Inverse Methods for Atmospheres: Theory and Practice, Singapore: World Scientific, 2008.

Rozanov, V. V. and Rozanov, A. A.: Relationship between different approaches to derive weighting functions related to atmospheric remote sensing problems, J. Quant. Spectr. Rad. Transfer, 105, 217-242, 2007.

Rozanov, V. V., Rozanov, A. V., Kokhanovsky, A. A., and Burrows, J. P.: Radiative transfer through terrestrial atmosphere and ocean: software package SCIATRAN, J. Quant. Spectr. Rad. Transfer, submitted, 2012.
Sobolev, V. V.: Light Scattering in Planetary Atmospheres. Oxford, England: Pergamon Press, 1975.

Ustinov, E.: Passive remote sensing of planetary atmospheres and retrievals of atmospheric macro- and microphysical parameters, J. Quant. Spectr. Rad. Transfer, 103, 217-230, 2007.

van de Hulst, H.: Light Scattering by Small Particles, New York : Dover, 1981.

Zhang Z., Platnick, S., Yang, P., Heidinger, A., and Comstock, J. M.: Effects of ice particle size vertical inhomogeneity on the passive remote sensing of ice clouds, J. Geophys. Res., 115, 4763, doi:10.1029/2010JD013835, 2010. 